【Short Paper】

\title{
A two-dimensional finite volume scheme solving the axisymmetric radiative heat transfer based on general structured grids
}

\author{
Jingying WANG*, Pengfei JU** and Li LEI* \\ * School of Energy and Power Engineering, Shandong University \\ No. 17923 Jingshi Road, Jinan, Shandong 250061, China \\ ** Tianjin Key Laboratory for Advanced Mechatronic System Design and Intelligent Control, Tianjin University of Technology \\ No. 391 Bin Shui Xi Dao Road, Xiqing District, Tianjin 300384, China \\ E-mail: jupengfei_tjut@126.com
}

Received: 20 December 2018; Revised: 1 February 2019; Accepted: 11 February 2019

\begin{abstract}
A two-dimensional finite volume method (FVM) based on structured grids is developed to solve the axisymmetric radiative heat transfer with absorbing, emitting and anisotropically scattering media. The present scheme is not derived by discretizing axisymmetric radiative transfer equation (RTE), but by taking the axisymmetric limit of general 3D radiation FVM, which can save the computation workloads greatly and avoid the singularity of the axisymmetric RTE when $r \longrightarrow 0$. Because of sharing same philosophy and meshes, it has very strong potentials of being coupled with popular computational fluid dynamics (CFD) solvers. This method is also fully validated by three benchmark cases: the cylindrical enclosure, truncated conical enclosure, and rocket nozzle, and performs excellently both in prediction accuracy and geometric flexibility.
\end{abstract}

Keywords : Finite volume method, Axisymmetric radiative heat transfer, Structured grids

\section{Introduction}

At present, FVM has been developed to solve radiation problems with advantages of allowing the complex geometry and full conservation by integrating RTE over each spatial control volume and solid angle (Raithby and Chui, 1990; Coelho, 2014; Sun et al., 2017). It also has very strong potentials of being coupled with many CFD solvers by sharing same philosophy and grids, such as Data Parallel Line Relaxation (DPLR) (Wright et al., 1998) and Parallel Hypersonic Aerothermodynamics and Radiation Optimized Solver (PHAROS) (Hao et al., 2017).

Axisymmetric radiative heat transfer is widely encountered in many engineering applications, such as boilers, furnaces, rocket nozzles, and even re-entry vehicles (Liu et al., 2000; Perez et al., 2004; Shang and Surzhikov, 2011). The axisymmetric approximation can save solving efforts greatly by simplifying a radiation problem from three-dimension (3D) into two-dimension (2D). Currently, axisymmetric radiative heat transfer is always calculated by two kinds of FVM schemes. One is directly to use a general 3D FVM to calculate the axisymmetric radiation with some artifices. Chui et al. (1992) used the 3D FVM with a mapping technique to predict the axisymmetric radiative transfer in cylindrical enclosures. Murthy and Mathur (1998) employed a conservative discretization of the angular redistribution term to extend 3D FVM to compute the axisymmetric radiation. Kumar and Eswaran (2013) adopted a general 3D FVM solver to compute the axisymmetric radiation problem by treating boundary conditions specially in the third direction. However, these approaches cannot reduce solving costs radically, because they still solve the axisymmetric radiative transfer as a 3D problem. The other is to develop a 2D FVM scheme based on the axisymmetric RTE. Kim and Eswaran (2010) integrated the axisymmetric RTE to deduce a FVM scheme with the particular directional weights to investigate the radiative transfer in an axisymmetric enclosure. However, this way needs to face difficulties in dealing with the singularity of the angular redistribution term when $r \longrightarrow 0$. To overcome above-mentioned drawbacks, Tian and Chiu (2005) proposed a 2D FVM to solve the axisymmetric radiative heat transfer by simplifying 
the general 3D FVM rather than discretizing the axisymmetric RTE. However, Tian et al's scheme can only be suitable for the Cartesian grids.

The objective of this study is to develop a new 2D FVM for solving the axisymmetric radiative heat transfer. The present scheme can be regarded as an extended version of Tian et al.'s scheme (Tian and Chiu, 2005), but not limited to using Cartesian grids. Because of sharing same philosophy and general 2D structured grids, the scheme is promisingly coupled with present popular CFD solvers. The method is also fully validated by three benchmark cases of different geometries and participating media.

\section{Methodology}

\subsection{FVM for RTE}

The RTE for gray participating media has the following form:

$\frac{\mathrm{d} I(\boldsymbol{r}, \boldsymbol{s})}{\mathrm{d} s}=-\beta I(\boldsymbol{r}, \boldsymbol{s})+\kappa I_{b}(\boldsymbol{r})+S(\boldsymbol{r}, \boldsymbol{s})$

and the source term is

$$
S(\boldsymbol{r}, \boldsymbol{s})=\frac{\sigma}{4 \pi} \int_{4 \pi} I\left(\boldsymbol{r}, \boldsymbol{s}^{\prime}\right) \Phi\left(\boldsymbol{s}^{\prime}, \boldsymbol{s}\right) \mathrm{d} \Omega^{\prime}
$$

where $I$ is the radiative intensity, $\boldsymbol{r}$ is the spatial position vector and $\boldsymbol{s}$ is the unit direction vector; $\kappa, \sigma$ and $\beta$ are the absorption, scattering and extinction coefficients, respectively, and $\beta=\kappa+\sigma ; \Phi$ is the scattering phase function depending on the incident direction vector $s$ and scattered direction vector $\boldsymbol{s}^{\prime} ; \Omega$ represents the solid angle, and the subscript ' $b$ ' represents the black body.

According to FVM principles and the divergence theorem (Raithby and Chui, 1990), over a control volume $\Delta V$ and solid angle $\Delta \Omega$, the equation (1) is integrated into

$$
\int_{\Delta \Omega} \int_{\partial V} I(\boldsymbol{r}, \boldsymbol{s})(\boldsymbol{s} \cdot \boldsymbol{n}) \mathrm{d} A \mathrm{~d} \Omega=\int_{\Delta \Omega} \int_{\Delta V}\left[-\beta I(\boldsymbol{r}, \boldsymbol{s})+\kappa I_{b}(\boldsymbol{r})+S(\boldsymbol{r}, \boldsymbol{s})\right] \mathrm{d} V \mathrm{~d} \Omega
$$

where $\partial V$ represents the surface of $\Delta V$ and $\boldsymbol{n}$ is the unit normal vector of $\partial V$ pointing outside $\Delta V$. The equation (3) is the general FVM formulation for RTE.

\subsection{Axisymmetric FVM}

The new 2D axisymmetric FVM scheme based on general structured grids is derived in the cylindrical coordinate system as shown in Fig.1. The unit direction vector $\boldsymbol{s}$ with angular coordinates $\{\theta, \varphi\}$ is expressed in Cartesian-bases $\{\boldsymbol{i}$, $\boldsymbol{j}, \boldsymbol{k}\}$ as

$$
\boldsymbol{s}=\cos \theta \cdot \boldsymbol{i}+\sin \theta \cos \varphi \cdot \boldsymbol{j}+\sin \theta \sin \varphi \cdot \boldsymbol{k}
$$

where $0 \leq \theta \leq \pi$ and $0 \leq \varphi<2 \pi$. Assume the whole $4 \pi$ solid angle is divided into $L \times M$ nonoverlapping solid angles $\Delta \Omega^{l, m}, l=1,2, \ldots, L, m=1,2, \ldots, M$, and the corresponding central direction is represented by angular coordinates $\left\{\theta^{l}\right.$, $\left.\varphi^{m}\right\}$. The foregoing division is equally discretized on both angular directions, and is fixed, even when the spatial position changes. Therefore, $\Delta \Omega^{l, m}$ follows:

$\Delta \Omega^{l, m}=\int_{\theta^{l}-\frac{1}{2} \Delta \theta}^{\theta^{l}+\frac{1}{2} \Delta \theta} \int_{\varphi^{m}-\frac{1}{2} \Delta \varphi}^{\varphi^{m}+\frac{1}{2} \Delta \varphi} \sin \theta \mathrm{d} \theta \mathrm{d} \varphi=2 \sin \theta^{l} \cdot \sin \frac{\Delta \theta}{2} \cdot \Delta \varphi$

where $\Delta \theta$ and $\Delta \varphi$ are angular intervals.

As shown in Fig.2, a hexahedral control volume with the centroid point $P$ forms by rotating a quadrangle around the $x$-axis from $\varphi_{0}=-\Delta \varphi_{0}$ to $\varphi_{0}=+\Delta \varphi_{0}$, and its projection on the plane $\varphi_{0}=0$ is marked as $a b c d$ (Fig.2(b)). This paper further defines $i e h l$ as the north face, $j f g k$ as the south face, iefj as the west face, lhgk as the east face, efgh as the top face, and $i j k l$ as the bottom face of the control volume. Additionally, $\boldsymbol{n}_{n}, \boldsymbol{n}_{s}, \boldsymbol{n}_{w}, \boldsymbol{n}_{e}, \boldsymbol{n}_{t}$, and $\boldsymbol{n}_{b}$ are the corresponding unit normal vector of each face, respectively. 


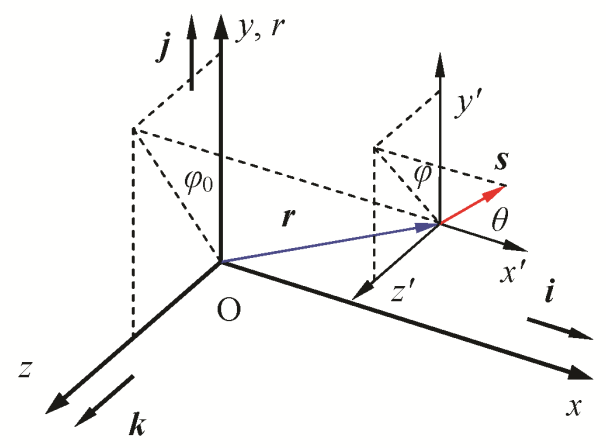

Fig. 1 Cylindrical coordinate system $\left\{x, r, \varphi_{0}\right\}$ and the unit direction vector $\boldsymbol{s}$.

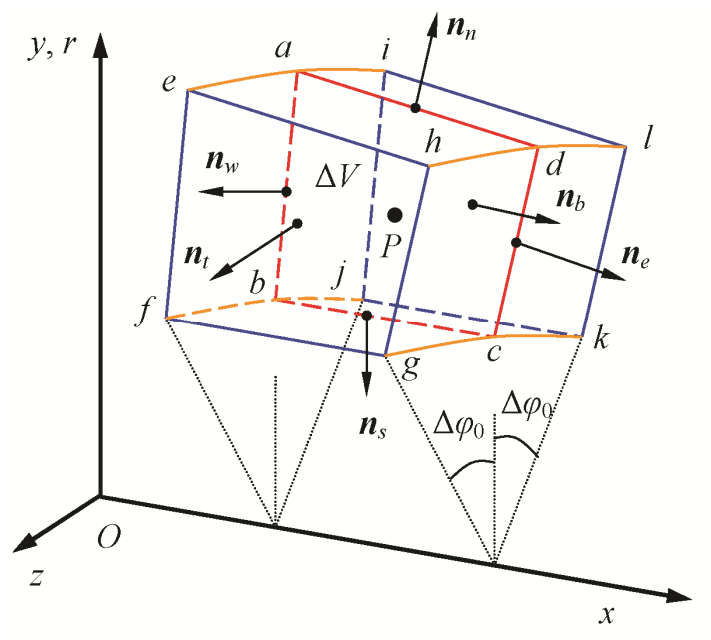

(a)

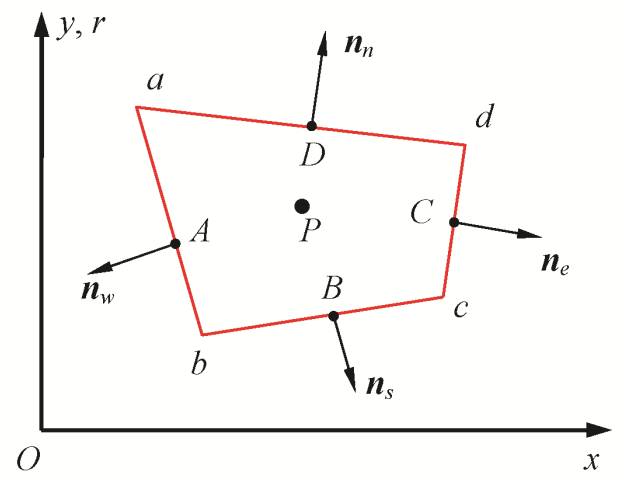

(b)

Fig. 2 Hexahedral control volume: (a) 3D control volume in the cylindrical system; (b) quadrangle $a b c d$ at $\varphi_{0}=0$.

For the control volume $\Delta V$ in Fig.2 and a fixed solid angle $\Delta \Omega^{l, m}$, the equation (3) is rewritten as follows:

$\sum_{i=n, s, w, e, t, b} \int_{\Delta \Omega^{l, n}} \int_{\partial V_{i}} I(\boldsymbol{r}, \boldsymbol{s})(\boldsymbol{s} \cdot \boldsymbol{n}) \mathrm{d} A \mathrm{~d} \Omega=\int_{\Delta \Omega^{\prime, m}} \int_{\Delta V}\left[-\beta I(\boldsymbol{r}, \boldsymbol{s})+\kappa I_{b}(\boldsymbol{r})+S(\boldsymbol{r}, \boldsymbol{s})\right] \mathrm{d} V \mathrm{~d} \Omega$

The radiative intensity on each face in the left-hand of Eq.(6) is approximated by the intensity at the face center, while the intensity in the control volume is replaced by the intensity at point $P$. Thus, there is the approximation as follows:

$\sum_{i=n, s, w, e, t, b} I_{i}^{l, m} A_{i} D_{i}^{l, m}=\left(-\beta I_{P}^{l, m}+\kappa I_{b P}+S_{P}^{l, m}\right) \Delta V \Delta \Omega^{l, m}$

and the source term can be discretized as follows:

$S_{P}^{l, m}=\frac{\sigma}{4 \pi} \sum_{p=1}^{L} \sum_{q=1}^{M} I_{P}^{p, q} \Phi\left(\boldsymbol{s}^{p, q}, \boldsymbol{s}^{l, m}\right) \Delta \Omega^{p, q}$

where

$D_{i}^{l, m}=\int_{\Delta \Omega^{l, m}}\left(\boldsymbol{s} \cdot \boldsymbol{n}_{i}\right) \mathrm{d} \Omega=\boldsymbol{n}_{i} \cdot\left(D_{0}^{l, m} \boldsymbol{i}+D_{1}^{l, m} \boldsymbol{j}+D_{2}^{l, m} \boldsymbol{k}\right), i=n, s, w, e, t, b$

and 


$$
\left\{\begin{array}{l}
D_{0}^{l, m}=\int_{\Delta \Omega^{l, m}} \cos \theta \mathrm{d} \Omega=\frac{1}{2} \sin 2 \theta^{l} \sin \Delta \theta \Delta \varphi \\
D_{1}^{l, m}=\int_{\Delta \Omega^{l, m}} \sin \theta \cos \varphi \mathrm{d} \Omega=\left(\Delta \theta-\cos 2 \theta^{l} \sin \Delta \theta\right) \cos \varphi^{m} \sin \frac{\Delta \varphi}{2} \\
D_{2}^{l, m}=\int_{\Delta \Omega^{l, m}} \sin \theta \sin \varphi \mathrm{d} \Omega=\left(\Delta \theta-\cos 2 \theta^{l} \sin \Delta \theta\right) \sin \varphi^{m} \sin \frac{\Delta \varphi}{2}
\end{array}\right.
$$

$A_{i}$ is the area of the $i$ face, and there are

$$
\left\{\begin{array}{l}
A_{n}=r_{D} \cdot 2 \Delta \varphi_{0} \cdot l_{a d}, A_{s}=r_{B} \cdot 2 \Delta \varphi_{0} \cdot l_{b c} \\
A_{w}=r_{A} \cdot 2 \Delta \varphi_{0} \cdot l_{a b}, A_{e}=r_{C} \cdot 2 \Delta \varphi_{0} \cdot l_{c d} \\
A_{t}=A_{b}=A_{P} \\
\Delta V=r_{P} \cdot 2 \Delta \varphi_{0} \cdot A_{P}
\end{array}\right.
$$

$A_{P}$ is the area of the panel $a b c d$. For the face $e f g h$ and $i j k l$, the unit normal vector $\boldsymbol{n}_{t}$ and $\boldsymbol{n}_{b}$ are

$\left\{\begin{array}{l}\boldsymbol{n}_{t}=-\sin \Delta \varphi_{0} \boldsymbol{j}+\cos \Delta \varphi_{0} \boldsymbol{k} \\ \boldsymbol{n}_{b}=-\sin \Delta \varphi_{0} \boldsymbol{j}-\cos \Delta \varphi_{0} \boldsymbol{k}\end{array}\right.$

Combining Eqs.(9) and (12), it is obtained as follows:

$$
\left\{\begin{array}{l}
D_{t}^{l, m}=-\sin \Delta \varphi_{0} D_{1}^{l, m}+\cos \Delta \varphi_{0} D_{2}^{l, m} \\
D_{b}^{l, m}=-\sin \Delta \varphi_{0} D_{1}^{l, m}-\cos \Delta \varphi_{0} D_{2}^{l, m}
\end{array}\right.
$$

Substituting Eqs.(11) and (13) into (7), there is

$$
\begin{aligned}
& 2 \Delta \varphi_{0}\left(I_{n}^{l, m} D_{n}^{l, m} \cdot r_{D} l_{a d}+I_{s}^{l, m} D_{s}^{l, m} \cdot r_{B} l_{b c}+I_{w}^{l, m} D_{w}^{l, m} \cdot r_{A} l_{a b}+I_{e}^{l, m} D_{e}^{l, m} \cdot r_{C} l_{c d}\right) \\
& +\left[-\sin \Delta \varphi_{0} D_{1}^{l, m}\left(I_{t}^{l, m}+I_{b}^{l, m}\right)+\cos \Delta \varphi_{0} D_{2}^{l, m}\left(I_{t}^{l, m}-I_{b}^{l, m}\right)\right] A_{P} \\
& =\left(-\beta I_{P}^{l, m}+\kappa I_{b P}+S_{P}^{l, m}\right) \Delta \Omega^{l, m} \cdot r_{P} A_{P} \cdot 2 \Delta \varphi_{0}
\end{aligned}
$$

As shown in Fig.3, for the direction $s^{l, m}$, because of the axisymmetry, there should be

$$
I_{t}^{l . m}=I_{t}\left(\theta^{l}, \varphi^{m}\right)=I_{P}\left(\theta^{l}, \varphi^{m}-\Delta \varphi_{0}\right)=I_{P}\left(\theta^{l}, \varphi^{m}\right)-\frac{\partial I_{P}\left(\theta^{l}, \varphi^{m}\right)}{\partial \varphi} \Delta \varphi_{0}+o\left(\Delta \varphi_{0}\right)=I_{P}^{l, m}-\frac{\partial I_{P}^{l, m}}{\partial \varphi} \Delta \varphi_{0}+o\left(\Delta \varphi_{0}\right)
$$

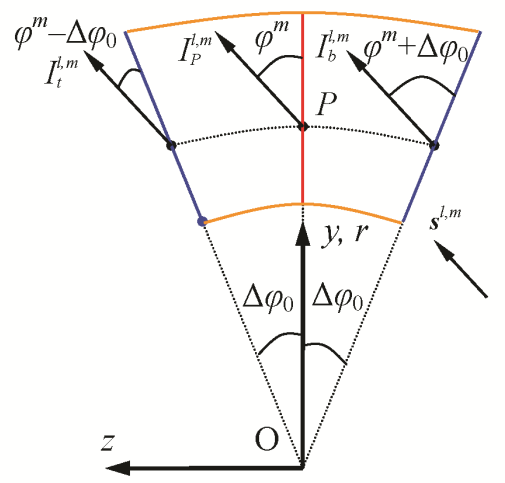

Fig. 3 Front view of the control volume. 
Similarly,

$I_{b}^{l, m}=I_{P}^{l, m}+\frac{\partial I_{P}^{l, m}}{\partial \varphi} \Delta \varphi_{0}+o\left(\Delta \varphi_{0}\right)$

Substituting Eqs.(15) and (16) into (14) and dividing the equation by $2 \Delta \varphi_{0}$, as $\Delta \varphi_{0} \rightarrow 0$, the final 2D FVM form of the axisymmetric radiative heat transfer is expressed as follows:

$$
\sum_{i=n, s, w, e} I_{i}^{l, m} D_{i}^{l, m} A_{i}^{\prime}-\left(I_{P}^{l, m} D_{1}^{l, m}+\frac{\partial I_{P}^{l, m}}{\partial \varphi} D_{2}^{l, m}\right) A_{P}=\left(-\beta I_{\mathrm{P}}^{l, m}+\kappa I_{b P}+S_{P}^{l, m}\right) \Delta \Omega^{l, m} \Delta V^{\prime}
$$

where

$$
\left\{\begin{array}{l}
A_{n}^{\prime}=r_{D} l_{a d}, A_{s}^{\prime}=r_{B} l_{b c} \\
A_{w}^{\prime}=r_{A} l_{a b}, A_{e}^{\prime}=r_{C} l_{c d} \\
\Delta V^{\prime}=r_{P} A_{P}
\end{array}\right.
$$

Obviously, the above scheme is suitable to solve the axisymmetric radiative transfer using general 2D structured grids and it can account for any absorbing, emitting and anisotropically scattering problems. Because of sharing same philosophy and structured grids, it is also prospective for the present 2D axisymmetric FVM scheme to be coupled with many CFD solvers smoothly. If the angular derivative of $I_{P}^{t m}$ in Eq.(17) is discretized by the central difference, it can be rewritten as follows:

$$
\sum_{i=n, s, w, e} I_{i}^{l, m} D_{i}^{l, m} A_{i}^{\prime}-\left(I_{P}^{l, m} D_{1}^{l, m}+\frac{I_{P}^{l, m+1}-I_{P}^{l, m-1}}{2 \Delta \varphi} D_{2}^{l, m}\right) A_{P}=\left(-\beta I_{P}^{l, m}+\kappa I_{b P}+S_{P}^{l, m}\right) \Delta \Omega^{l, m} \Delta V^{\prime}
$$

Assuming all boundaries are gray-diffuse walls, the ingoing $\left(\boldsymbol{s}^{l, m} \cdot \boldsymbol{n}_{w}<0\right)$ intensity $I_{w}^{l, m}$ at the wall can be given by

$$
I_{w}^{l, m}=\frac{\varepsilon_{w} \cdot \sigma T_{w}^{4}}{\pi}+\frac{\left(1-\varepsilon_{w}\right)}{\pi} \cdot \sum_{s^{p, a \cdot} \cdot n_{w}>0} I_{w}^{p, q}\left|\boldsymbol{S}^{p, q} \cdot \boldsymbol{n}_{w}\right| \Delta \Omega^{p, q}
$$

where $\boldsymbol{n}_{w}$ is the unit normal vector of the wall pointing outside the domain; $\varepsilon_{w}$ is the wall emissivity, and $T_{w}$ is the wall temperature; $\sigma$ is the Stefan-Boltzmann constant. The other directional intensity is interpolated from the domain inside.

In fact, for axisymmetry, Eq.(19) only needs to be solved in the half of the azimuthal space, that is $0 \leq \varphi \leq \pi$. In this paper, the step scheme (Tian and Chiu, 2005) is used for the spatial discretization of the radiative intensity at each control face to solve Eq.(19).

\section{Results}

\subsection{Cylindrical Enclosure}

The first benchmark case is a cylindrical enclosure with the absorbing, emitting and nonscattering medium, and its exact solution is available (Dua and Cheng, 1975). As shown in Fig. 4, the aspect ratio of the enclosure, $L / R$, is 2 . The medium in the enclosure is isothermal with the temperature $T_{0}$ and all bounded walls are black with the temperature $T_{w}$ $=0 \mathrm{~K}$. The grids are intentionally set to be nonorthogonal. Three situations with different optical thicknesses, $\tau_{R}=\kappa R=$ $0.1,1.0$, and 5.0 are calculated. Figure 5 compares the present numerical results of the radiative heat fluxes along the top wall with the exact solutions. The maximum errors for $\tau_{R}=0.1,1.0$, and 5.0 are $1.8 \%, 3.4 \%$ and $4.1 \%$, respectively, which are almost of the same magnitude as reported in previous literatures (Murthy and Mathur, 1998; Tian and Chiu, 2005).

\subsection{Truncated Conical Enclosure}

The geometry and grids of the conical enclosure with inclined angle $30^{\circ}$ is shown in Fig. 6 . The left side wall is hot with the temperature $100 \mathrm{~K}$, others are cold with the temperature $0 \mathrm{~K}$, and all walls are black. The medium in the enclosure is absorbing, emitting and isotropically scattering with an extinction coefficient $\beta=1.0 \mathrm{~m}^{-1}$. The cases of different scattering albedo, $\omega_{0}=\sigma / \beta$, varying from 0.0 to 1.0 , are calculated by the present scheme. Figure 7 shows the 
nondimensional radiative heat flux distribution along the left side wall, and the present results are greatly consistent with the solutions reported in the papers of Kim et al. (2010), Kim and Baek (2005).

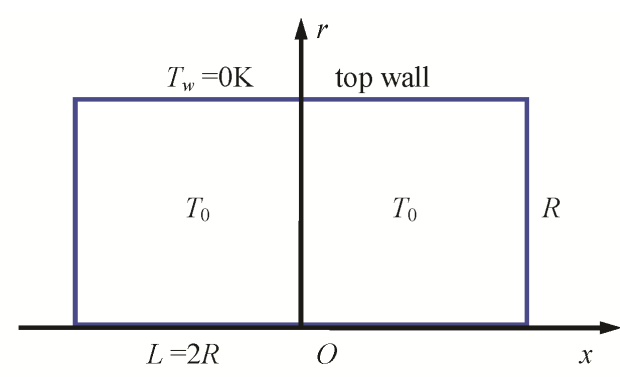

(a)

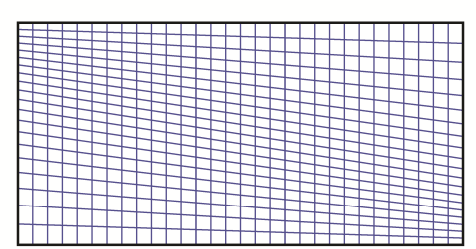

(b)

Fig. 4 Cylindrical enclosure: (a) schematic; (b) grids.

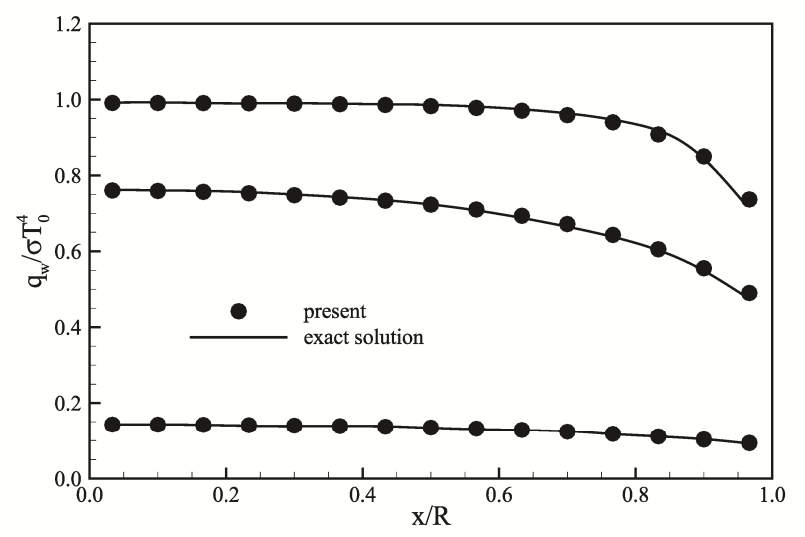

Fig. 5 Radiative heat flux on the top wall of the cylindrical enclosure.

\subsection{Rocket Nozzle}

A rocket nozzle which is considered to be a practical case is also computed. The geometry of the rocket nozzle model is depicted in Fig.8. The axial temperature distributions of nozzle wall and combustion products are listed in the paper of Nunes et al. (2000). The nozzle wall is gray with the emissivity of 0.8 ; the top boundary of the combustion chamber perpendicular to the $x$-axis is set to be black with the same temperature of the neighboring gas; the exiting cross-section is assumed to be gray with the emissivity of 0.8 and the temperature equal to the adjacent gas. The combustion products are treated as the absorbing, emitting and anisotropically scattering soot/carbon particles with the uniform diameter of $30 \mu \mathrm{m}$ and concentration $N_{p}=5 \times 10^{8} \mathrm{par} / \mathrm{m}^{3}$. The radiation properties of particles are calculated by the expressions of Nunes et al. (2000). Figure 9 illustrates the nondimensional radiative heat flux distribution along the nozzle wall. The present results show the good agreement with data reported in the papers of Nunes et al. (2000) and Salah et al. (2006).

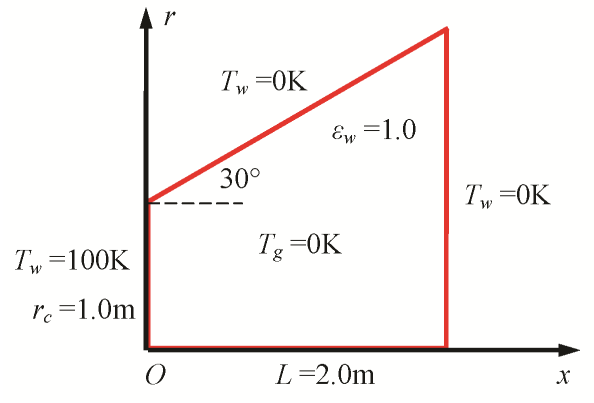

(a)

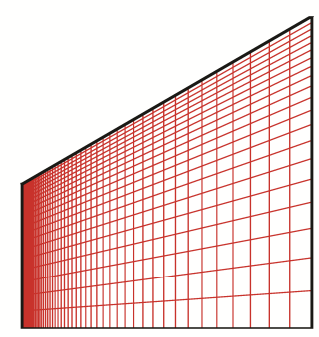

(b)

Fig. 6 Truncated conical enclosure: (a) schematic; (b) grids. 


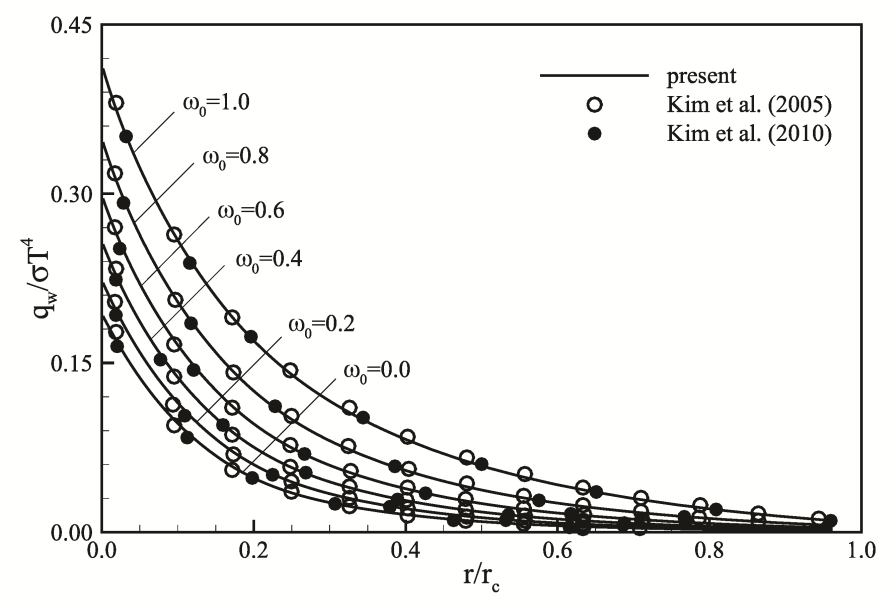

Fig. 7 The radiative heat flux distribution along the left side wall of the conical enclosure.

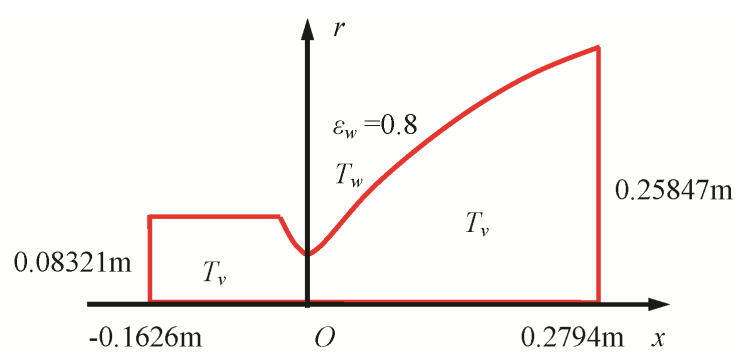

(a)

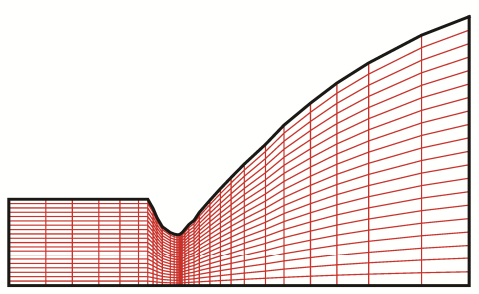

(b)

Fig. 8 The rocket nozzle: (a) schematic; (b) grids.

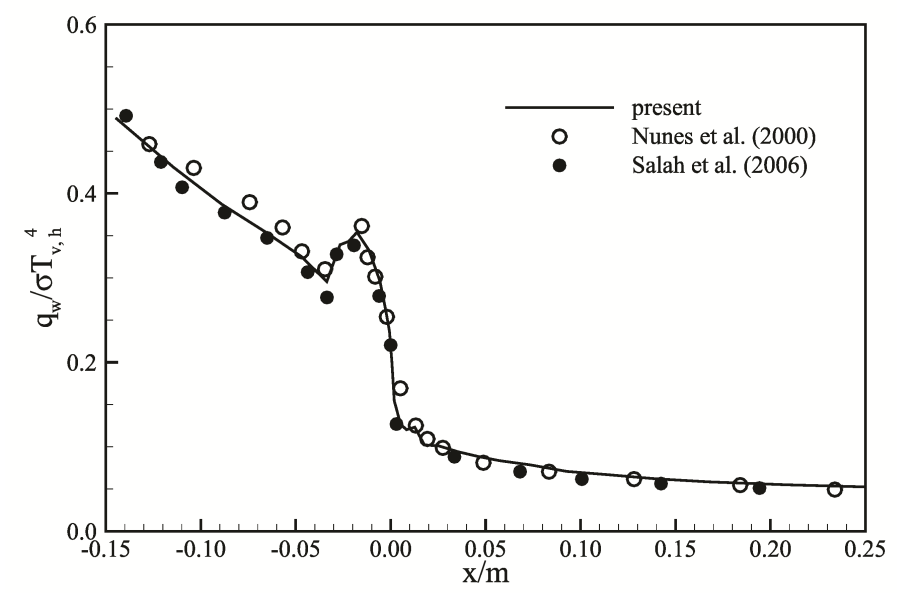

Fig. 9 Nondimensional radiative heat flux distribution along the nozzle axis.

\section{Conclusions}

A 2D FVM scheme solving the axisymmetric radiative transfer is obtained by taking the azimuthal limit of the 3D FVM, but not based on the direct discretization of the axisymmetric RTE. The scheme can be considered as the extended version of the Tian et al.'s scheme from Cartesian girds to general structured grids. Owing to sharing same philosophy and grids, the method has strong potentials of being coupled with popular CFD solvers. Compared with exact solutions and numerical data reported in literatures, the present scheme behaves very well both in prediction accuracy and geometric flexibility. 


\section{Fundings}

This work was supported by the China Postdoctoral Science Foundation Funded Project (No. 2016M602134); the Shandong Provincial Natural Science Foundation, China (No. ZR2016AB06); and the Fundamental Research Funds of Shandong University (No. 2016GN018).

\section{References}

Chui, E. H., Raithy, G. D. and Hughes, P. M. J., Prediction of radiative transfer in cylindrical enclosures with the finite volume method, Journal of Thermophysics and Heat Transfer, Vol. 6, No. 4 (1992), pp.605-611.

Coelho, P. J., Advances in the discrete ordinates and finite volume methods for the solution of radiative heat transfer problems in participating media, Journal of Quantitative Spectroscopy and Radiative Transfer, Vol. 145 (2014), pp.121-146.

Dua, S. S. and Cheng, P., Multi-dimensional radiative transfer in non-isothermal cylindrical media with non-isothermal bounding walls, International Journal of Heat and Mass Transfer, Vol. 18 (1975), pp.245-259.

Hao, J., Wang, J. and Lee, C., Development of a Navier-Stokes code for hypersonic nonequilibrium simulations, $21 \mathrm{st}$ AIAA International Space Planes and Hypersonics Technologies Conference (2017), Paper No. AIAA-2017-2164.

Kim, C., Kim, M. Y., Yu, M. J. and Mishra, S. C., Unstructured polygonal finite-volume solutions of radiative heat transfer in a complex axisymmetric enclosure, Numerical Heat Transfer, Part B: Fundamentals, Vol. 57, No. 3 (2010), pp.227-239.

Kim, M. Y. and Baek, S. W., Modeling of radiative heat transfer in an axisymmetric cylindrical enclosure with participating medium, Journal of Quantitative Spectroscopy and Radiative Transfer, Vol. 90 (2005), pp.377-388.

Kumar, P. and Eswaran, V., A methodology to solve 2D and axisymmetric radiative transfer problems using a general 3D solver, Journal of Heat Transfer, Vol. 135, No. 12 (2013), 124501.

Liu, J., Shang, H. M. and Chen, Y. S., Development of an unstructured radiation model applicable for two-dimensional planar, axisymmetric, and three-dimensional geometries, Journal of Quantitative Spectroscopy and Radiative Transfer, Vol. 66 (2000), pp.17-33.

Murthy, J. Y. and Mathur, S. R., Radiative heat transfer in axisymmetric geometries using an unstructured finite-volume method, Numerical Heat Transfer, Part B: Fundamentals, Vol. 33, No. 4 (1998), pp.397-416.

Nunes, E. M., Modi, V. and Naraghi, M. H. N., Radiative transfer in arbitrarily-shaped axisymmetric enclosures with anisotropic scattering media, International Journal of Heat and Mass Transfer, Vol.43, No.18 (2000), pp.3275-3285.

Perez, P., El Hafi, M., Coelho, P. J. and Founier, R., Accurate solutions for radiative heat transfer in two-dimensional axisymmetric enclosures with gas radiation and reflective surfaces, Numerical Heat Transfer Part B: Fundamentals, Vol. 47, No. 1 (2004), pp.39-63.

Raithby, G. D. and Chui, E. H., A finite-volume method for predicting a radiant heat transfer in enclosures with participating media, Journal of Heat Transfer, Vol. 112, No. 1 (1990), pp.415-423.

Salah, M. B., Askri, F., Jemni, A. and Nasrallah, S. B., Numerical analyses of radiative heat transfer in any arbitrarily-shaped axisymmetric enclosures, Journal of Quantitative Spectroscopy and Radiative Transfer, Vol. 97 (2006), pp.395-414.

Shang, J. S. and Surzhikov, S. T., Simulating stardust earth reentry with radiation heat transfer, Journal of Spacecraft and Rockets, Vol. 48, No. 3 (2011), pp.385-396.

Sun, Y., Zhang, X. and Howell, J. R., Assessment of different radiative transfer equation solvers for combined natural convection and radiation heat transfer problems, Journal of Quantitative Spectroscopy and Radiative Transfer, Vol. 194 (2017), pp.31-46.

Tian, W. and Chiu, W. K. S., A two-dimensional scheme for axisymmetric radiative heat transfer using the finite-volume method, Numerical Heat Transfer, Part B: Fundamentals, Vol. 47, No. 3 (2005), pp.199-211.

Wright, M. J., Candler, G. V. and Bose, D., Data-parallel line relaxation method for the Navier-Stokes equations, AIAA Journal, Vol. 36, No. 9 (1998), pp.1603-1609. 